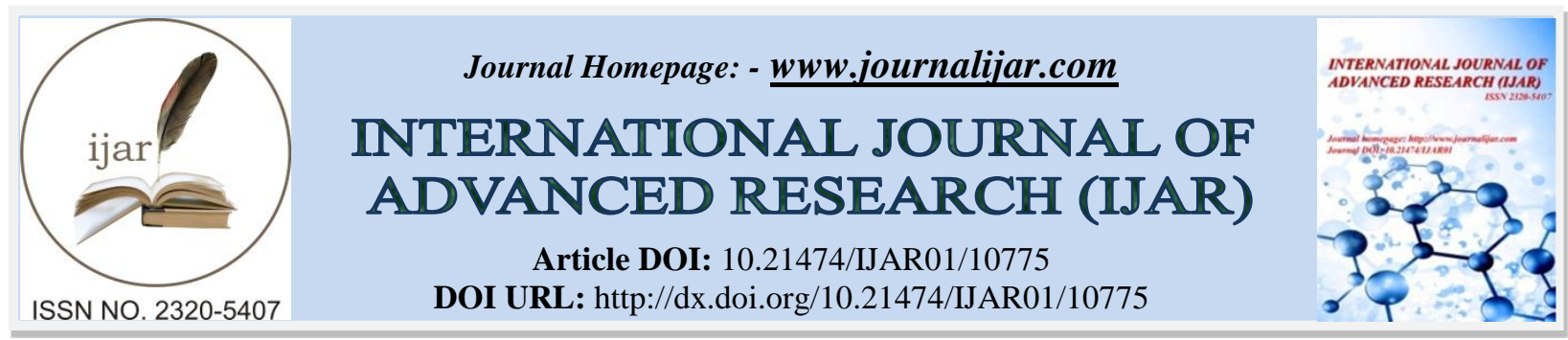

RESEARCH ARTICLE

\title{
THE SUITABILITY OF THE EGYPTIAN BALADI SHEEP (OVIS ARIES) AS A LARGE ANIMAL MODEL FOR TEMPOROMANDIBULAR JOINT STUDIES
}

\author{
Enas El-Hady
}

Department of Anatomy and Embryology, Faculty of Veterinary Medicine. Zagazig University, 44511, Egypt.

\section{Manuscript Info}

Manuscript History

Received: 10 February 2020

Final Accepted: 12 March 2020

Published: April 2020

Key words:-

Sheep, TMJ, Animal models, Anatomy,

Histology, SEM

\begin{abstract}
This study aimed to characterize and evaluate normal temporomandibular joint (TMJ) of the Egyptian Baladi sheep anatomically and histologically as a large animal model. Fifteen heads of adult apparently healthy male sheep (Ovis aries) were utilized for; macro- and microscopical examination. Also, bony preparations, radiology, computed tomography and scanning electron microscopy were achieved. The TMJ consisted of mandibular condyle of the mandible, glenoid fossa of temporal bone and articular disc in-between. The mandibular condyle was flattened laterally, convex medially and slopped caudomedially. The glenoid fossa was elliptical in shape, concavoconvex from backward to forward. The disc was a smooth, elliptical plate, biconcave, thin centrally and thick peripherally with sloped end caudomedially. Histologically, the fibrocartilage layer of the condyle and glenoid fossa was differentiated into four zones; fibrous, proliferative, hypertrophic, and calcification zones. The fibrocartilagenous disc consisted of thick densely packed bundles of collagen fibers with chondrocytes in-between. The complex architecture of articular disc fibers was revealed by light and scanning electron microscopic examination. The collagen fibers orientation was perpendicular in the anterior and posterior regions, and parallel anteroposterior in the central region. There were many anatomical similarities between sheep and human TMJ in; relations, size, shape, position of the disc, histological structure and surgical approach. Furthermore, the sheep are more economic, available by large numbers, high ethical suitability, all this renders the sheep not only suitable but ideal choice as a large animal model over other animal models.
\end{abstract}

Copy Right, IJAR, 2020,. All rights reserved.

\section{Introduction:-}

TMJ is a particular diarthroidal joint, essential for most of mammals' functional jaw, that unique to mammals and differentiates them from other vertebrates (Smith, 2001). TMJ is very important for normal mouth functions; such as mastication, speech, deglutition, yawning and snoring (Ingawalé and Goswami, 2009). These functions were achieved by the assistance of a specialized group of strong muscles that control the jaw movement (Porto et al., 2010).

Corresponding Author:- Enas El-Hady

Address:- Department of Anatomy and Embryology, Faculty of Veterinary Medicine. Zagazig

University, 44511, Egypt. 
The essential components of TMJ are mandibular condyle of the mandible, glenoid fossa of the temporal bone, disc in-between them and synovial fluid (de Moraes et al., 2011 and Purcell et al., 2012).

The articular disc has vital roles as, recompensing the incongruence of the articular surfaces (Piette, 1993), scattering and allocating the intrarticular loads (Dolwick, 1995 and Tanaka and van Eijden, 2003) and stabilizing the joints during translation by its thick rim which has special viscoelastic properties (Piette, 1993 and Dolwick, 1995).

The repair of bone and cartilage disorders in sheep is similar to human, so the selection of animal model is the key in these trials to compare the biological modifications with man disease (Ishimaru and Goss, 1992). Sheep and goats are suitable as TMJ models because their deficiency in protection against anteroposterior forces, posterior joint function and the translator ability of the joint. They are abundant, cheap, and easily maintained and controlled (Bifano et al., 1994). In the last decades, many authors use the sheep as a model in TMJ researches as a result of the aforementioned causes.

Taking into consideration that there are little reviewed literature publications on the detailed anatomy of sheep TMJ. Thus, the aim of this work is to give a full explanation of the morphology of TMJ of normal sheep via gross and microscopic examination, bony preparations, radiology, computed tomography (CT) and scanning electron microscopy (SEM). Also to compare TMJ of normal sheep with that of the human and other animal models to evaluate the possibility of using it as a model in TMJ researches.

\section{Material and Methods:- \\ Ethical approval:}

The handling of animals in this work followed the guidelines of the Institutional Animal Care and the Research Ethics Committee of the Zagazig University, with an ethical approval number of ZU-IACUC/2/F/188/2019.

\section{Animals used:}

This work was carried out on (15) heads of adult (20-24 month old), apparently healthy male sheep without any history of TMJ disorders. The animals were slaughtered in Zagazig abattoir, the heads of which were obtained immediately after slaughtering.

\section{Macroscopical examination:}

Six heads were used; five of them were dissected starting from the skin till reaching the joint cavity to elucidate all anatomical structures associated with the TMJ.Then the discs were separated and their dimensions were taken. The sixth head was used to clarify the accurate site of intrarticular injection of TMJ. Then the same head was cut sagittally to reveal the compartments of the TMJ.

\section{Bony preparations:}

Two heads were used; all soft tissues were removed as it was possible with surgical instruments. The closely adherent residual tissues were detached by boiling the skulls in water, then bleaching with $30 \% \mathrm{H} 2 \mathrm{O} 2$ solution (Onwuama et al., 2012).

\section{Radiological examination:}

Dorsolateral views were taken from a head at $40 \mathrm{KV}$ and $10 \mathrm{mAs}$ in the department of surgery, Faculty of Veterinary Medicine, Zagazig University.

\section{Computed tomography (CT):}

CT was performed on one head at (120 kV-130 mA) in AL-Bayan Center in Belbes, Sharkia Governorate, Egypt.

\section{Histological examination:}

TMJ complexes of three fresh heads were removed en bloc with a band saw and fixed with $10 \%$ natural buffered formalin for three days. All specimens were decalcified in buffered 19\% Ethylene di-amine tetra-acetic Acid (EDTA) ( $\mathrm{pH}$ 7.2-7.4) for 15- 20 days at room temperature. The decalcified specimens were embedded in paraffin. Five- $\mu$ m-thick sections were obtained and stained with Hematoxylin and eosin, and Crossman-Trichrome stains (Bancroft, 2008). 


\section{Scanning electron microscopy (SEM):}

TMJ discs of two fresh heads were separated immediately after slaughter. Then they were fixed with $2.5 \%$ of gluteraldehyde solution for 48 hours. After that they were washed by $0.1 \mathrm{M}$ phosphate buffer solution twice. The tissues were kept in a $1 \%$ of osmium tetra-oxide solution for one hour, then handled by acetone sequences and dehydrated in ethanol and drying in a critical point dryer. The specimens were coated by Gold-Palladium and studied by a scanning electron microscope at various magnifications. The specimens were examined with JSM6510LV scanning electron microscope (JEOL CO., USA) at an accelerating voltage of 20, $30 \mathrm{kV}$. This section was performed at Faculty of Agriculture, Mansoura University.

All the observed results illustrated by Photographs were taken by Canon PowerShot A3300IS digital camera.

\section{Results:-}

By gross morphological, CT and radiological examinations, there were no qualitative differences between the right and left joints of the same animal. The TMJ located rostral to the external acoustic meatus just caudal to the posterior end of the zygomatic arch. It consisted of mandibular condyle of the mandible, glenoid fossa of the temporal bone and articular disc in-between (Figs. 1 and 2).

The mandibular Condyle had a smooth, whitish, glistening articulating surface. It was flattened laterally, convex medially and slopped caudomedially. It was separated by mandibular notch from the coronoid process. An elongated fovea was present on the anteromedial aspect of the condyle for the attachment of the lateral pterygoid muscle (Fig.1A-E and Fig. 2A,B,G,H).

The glenoid fossa formed the base of the skull in which the mandibular condyle articulated. The articular surface of the fossa was much larger than that of the condyle. It was elliptical in shape, concavoconvex from backward to forward. It was bounded posteriorly by a small elevation termed retroarticular process (postglenoid process) which was larger medially. The oval foramen located medial to the fossa (Fig. 1 and Fig.2A). The mandibular condyle and the temporal surface of the glenoid fossa were wider lateromedially than rostrocaudally (Fig. 1F and Fig.2B).

The articular disc divided the joint cavity into larger superior and smaller inferior partitions. It was attached peripherally to the articular capsule and the margins of the articular surfaces. The disc divided anteriorly into upper and lower band attached to the temporal bone, the mandibular condyle respectively and the lateral pterygoid muscle fibers in-between. Posteriorly, the disc is divided into inferior and superior lamellae.The inferior lamella was thin, extended from fibrous disc to the posterior surface of head of condyle (Fig.1C-E and Fig. 2A,H). The disc was a smooth, white biconcave elliptical plate acted as a mobile fossa for the condyle. It had two surfaces; dorsal and ventral. The former one was thin concave centrally and thick at peripheral borders with sloped end caudomedially. The ventral surface was slightly concave along its length. It was measured about $23.83 \pm 1.5 \mathrm{~mm}$, lateromedially and $17.28 \pm 0.6 \mathrm{~mm}$ rostrocaudally (Fig.2C,D).

The articular capsule was relatively wide and attached to the margins of the articular surfaces (glenoid fossa and mandibular condyle). Anteriorly, it was attached to the zygomatic process of the malar bone and to zygomatic process of temporal bone posteriorly. Medially the capsule attached to the medial boundary of the glenoid fossa superiorly and the medial aspect of the neck of mandible inferiorly. The articular capsule was rich with blood vessels anteriorly than posteriorly. Laterally, it was reinforced by the lateral ligament, which restrains dislocation of the condyle (Figs.1C, D and 2G).

The accurate site of intra-articular injection of TMJ was about $1 \pm 0.25 \mathrm{~cm}$ rostral to the external ear (Fig. 2E).

The steps of dissection for surgical approach of TMJ, firstly, after removal of the skin and subcutaneous tissue, the masseter muscle appeared attached dorsally to the zygomatic arch and ventrally to the lower border and angle of mandible. By removing the layers of masseter muscle the components of TMJ can be observed. The condylar head was situated just caudal to the posterior end of zygomatic arch, the glenoid fossa noticed by reflecting the arch and detaching the condyle. The TMJ cavity was clearly seen as superior and inferior cavities separated by the interarticular disc. (Figs.2F-H).

Microscopically TMJ articular surfaces (mandibular condyle and glenoid fossa) were covered by a fibrocartilaginous layer instead of hyaline cartilage. The fibrocartilage layer was arranged into four zones from outside to inside; the superficial (fibrous), transitional (proliferative), deep (hypertrophic), and calcified (calcification) zones (Fig. 3A-D). 
The fibrous zone was composed of compact collagen fibers with few flattened chondrocytes. The proliferative zone was consisted of condensed spherical chondrocytes surrounded by thick collagen matrix, the cell density was the highest among the four layers. The hypertrophic zone formed from large chondrocytes arranged in the form of columns up right to the articular surface. The calcified zone was in the form of round chondrocytes situated in uncalcified lacunae with presence of blood capillaries. The orientation of collagen fibrils was parallel in the fibrous zone, haphazardly in proliferative zone and perpendicular in hypertrophic zone and calcified zone. The glenoid fossa fibrocartilaginous layer was similar but thinner than that of mandibular condyle. The thickness of fibrocartilaginous layer varies according to its site, it was thinner at the center than at either sides (Fig. 3B-D). The cancellous bone under the calcified zone of the mandibular condyle appeared in the form of variable sized irregular trabeculae intersecting with each other, their size, gradually increased at the center, (Fig. 3E). The glenoid fossa had both types of bones; thick compact cortical bone externally and widely spaced cancellous bone deeply (Fig. 3F).

The articular disc was fibrocartilaginous, appeared thin at the center and wide at both ends. The anterior and posterior parts of the disc were consisted of thick bundles of collagen fibers randomly arranged and tightly packed. Chondrocytes were existed in lacunae present in clusters in the anterior part or scattered in-between less dense collagen fibers in the rest of the disc (Fig. 3G,H,I,J). The thin central part of the disc composed of densely packed collagen fibers formed thin bundles had undulating course in an anteroposterior orientation with dispersed fibroblasts in-between (Fig. 3L). Few small diameter blood vessels were observed in-between the collagen fibers in the anterior part of the disc. The disc attached anteriorly with loose connective tissue contained large sized blood vessels and nerves (Fig. $3 \mathrm{~K}, \mathrm{M}$ ).

The articular capsule was observed as a delicate folded membrane of collagenous tissue widened at the posterior part. It was related anteriorly to the lateral pterygoid muscle.Variable sized vessels were noticed in the loose connective tissue anterior to the joint (Fig 3M).

By SEM the inner face of synovial membrane that line the articular capsule appeared folded under scanning electron microscope with variable sized blood vessels anteriorly. The superior and inferior joint cavities were noticed inbetween the disc and articular capsule. The latter padded with rounded cells arranged in clusters like bunches of grape (Fig. 4A-E).

The architecture of TMJ disc fibers was composite. The dorsal surface of the disc appeared as corrugated folds. The degree of crimping was clear anteriorly and posteriorly than in the center. The collagen fibers of the disc were arranged in perpendicular interrupting pattern in the anterior and posterior regions, the fibers had a parallel anteroposterior orientation in the central region. The fiber orientation in the rostral and caudal bands was lateromedial (Fig. 4F-H). The collagen fibers thickness was apparently thinner in the central than anterior and posterior regions. Chondrocytes were observed in-between the fibers in all regions. Erythrocytes were also noted inside the blood vessel in the anterior region. Elastic fibers were seemed across all regions, with a gradual increase from anterior to posterior (form networks) (Fig. 4I-N).

\section{Discussion:-}

Although no animal model can entirely emulate human circumstances, animal models are vital for pathogenesis assessment, novel technologies trying and new procedures application. Various animal models have been tried for TMJ studies; small animals (rodents and rabbits) were usually used, but there are clear variations in size, anatomy and function of the TMJ from humans. Although, primates were greatly similar to humans, but they have difficulties relating to the ethics of their testing and high cost. Conversely, the sheep TMJ is actually equivalent to human TMJ adding to its low cost and high ethical suitability (Bosanquet and Goss, 1987). Contrary to the farm pig and minipig, the ease of surgical approach to TMJ and specimen accessibility of sheep and goat construct them to be more preferable as large animal models (Almarza et al., 2018and Almarza and Chung, 2019).

TMJ of sheep is a bi-condylar joint that connects the mandible to the skull and controls jaw movement. Sheep, goats and cattle are closely related ruminant artiodactyls have identical TMJs. As they are herbivores, they have a distinctive jaw apparatus and TMJs are specialized for great mobility in the transverse plane (Herring, 2003). From this point Bermejo et al. (1993) decided that, as the pig is the only omnivore moreover its disc was similar to human, it was the most ideal animal model when compared with dogs, cats, rabbits, rats, cows, sheep, and goats. 
The mandibular condyle was wider lateromedially than rostrocaudally, (Ângelo et al., 2016) in Black Merino sheep. We revealed that, not only the articular surfaces of TMJ were wider lateromedially, but also the glenoid fossa was much larger than the mandibular condyle, so as to permit free movement of the condylar head mediolaterally for grinding of food. The mandibular condyle was flattened laterally, convex medially and slopped caudomedially unlike that of human which was rounded and convex. On the other hand, mandibular condyle was mediolaterally concave in Black Merino sheep (Ângelo et al., 2016) and roe deer (Kabak et al., 2014).

Our results clarified that, the articulating surfaces of the TMJ were greatly incongruent. So, the contact areas of the opposing articular surfaces were very small. The presence of a fibrocartilaginous disc in-between is believed to distribute masticatory forces preventing the injury of articular cartilage and inhibiting their wear by preventing the contact between them (Tanne et al., 1991; Scapino et al., 1996; Chin et al., 1996 and Tanaka et al., 2008). Also, the disc acts as a pillow for pressure absorption and easy movement of the condyle during opening and closing the mouth (Ide et al., 1991). The joint cavity was divided by the disc into two partitions (superior and inferior) (Ide et al., 1991 and Tanaka et al., 2008). The disc was thin centrally and thick peripherally connected to the joint capsule, mandibular head and temporal bone. These results were on the same line with that observed in Akkaraman sheep (Kabak, 2002) and horse (Rodriguez et al., 2006). Regarding the dimensions of the articular disc of the sheep, it was more similar to that of humans with regard to other animal models. These results were in harmony with Kalpakci et al. (2011) in pig and Kabak et al. (2014) in roe deer.

The most compatible site for intra-articular interference was the caudolateral part of the joint about $1 \pm 0.25 \mathrm{~cm}$ rostral to the external ear due to the location of postglenoid process; similar findings were recorded in the roe deer (Kabak et al., 2014).

Interestingly, the surgical approach of TMJ in sheep was easy; via direct lateral preauricular access resembling human (Ness, 2011). This entrance cannot be achieved in minipigs (Vapniarsky et al., 2017) and pig (Almarza, et al., 2018) as the lateral approach to TMJ was disallowed by the zygomatic arch. Since the intrusive arthroscopic surgical procedures of the TMJ strictly need the acquirement of sufficient skills that can hardly be acquired from the patients alone. The anatomical relations of TMJ with the external acoustic meatus, foramen ovale and position of the disc are comparable to human TMJ. So, the sheep TMJ could serve as trusty models for education and enhancement of arthroscopic surgery in the TMJ (Bosanquet et al., 1991; Ogi et al., 1999 and Ângelo et al., 2016).

Regarding our histological findings of TMJ articular surfaces resembled the previous studies; they were characterized by fibrocartilaginous covering instead of hyaline cartilage (Ide et al., 1991). This fibrocartilaginous layer was differentiated into four zones, similar results were observed by (Cornish, 2005; Yan et al., 2013 and Ângeloet al., 2016) in sheep and (Rashed, 2015) in dog. On the other hand, Tahmasebi-Sarvestani (1997) in Australian Merino sheep revealed three layers and Lin et al. (2018) in dog mentioned only two layers (a compact fibrous connective tissue and subchondral bone). The variation in thickness of the cartilagenous cover of TMJ indicated that the highest loading area of the TMJ was thinner in the center (Ângelo et al., 2016) in sheep. Chondrocytes vary in shape, number, and size depending on the location of the articular cartilage. They are round or polygonal in the proliferative zone but flattened in the superficial zone (Jung, 2014). Our results clarified the presence of blood capillaries in the calcified zone. In contrast, Rashed (2015) revealed many capillaries in the fibrous zone in dog. Concerning the bone of the mandibular condyle, it was cancellous while the glenoid fossa had both cancellous and cortical bones. In the same line with Rashed (2015) in dog's glenoid fossa but she revealed little compact bone might be present in the condyle. On the other hand Cornish (2005) and Ângelo et al. (2016) in sheep revealed that the mandible contained both cancellous and compact bones, the first author added that the cancellous bone was in the form of plates and rods.

The fibrocartilaginous structure of the articular disc of the sheep, agree with the results of (Gillbe, 1973 and Kabak, 2002). Chondrocytes distribution within the disc differs according to site; they were in the form of clusters anteriorly or dispersed all over the disc. These findings were dissimilar to that of Kabak et al., (2014) in roe deer, where they were aligned in binary or triple isogene groups. The collagen bundles arrangement generally had a lateromedial course in the articular disc of roe deer (Kabak et al., 2014). The anterior and posterior bands feature dense interwoven collagen fibers oriented predominantly mediolaterally, whereas the crimped collagen fibers of the intermediate zone run anteroposteriorly (Sindelar et al., 2002). Respecting the location of sinusoid type blood vessels within the disc, we observed small sized blood vessels by light and SEM in the rostral part, similar findings were reported in sheep (Gillbe, 1973; Bosanquet and Goss, 1987) and roe deer (Kabak et al., 2014).On the other 
hand, these blood vessels were existent all over the disc in Akkaraman sheep (Kabak, 2002), Black Merino sheep (Ângelo et al., 2016) and human (Sabú et al. 2006), in the posterior part in goat (Bifanoet al., 1994), at the periphery in dog (Lin et al., 2018) and in the central part in Scandinavian moose (Ström et al., 1996). The fibrillary architecture of the articular disc was longitudinal in the superior and inferior regions; transversal and oblique in the central region (Minarelli et al., 1997and Sabú et al. 2006) in human. Kalpakci et al.(2011) in pig and Vapniarsky et al.(2017) in minipig revealed that collagen fibers orientation was anteroposterior in the central, lateral, and medial regions, and mediolateral in the posterior band. Lin et al. (2018) in dog noticed anteroposterior orientation of fibers in the central regions and lateromedial orientation in the rostral and caudal bands.

The collagen fibers were thinner in the central than anterior and posterior regions, on the same line, Lin et al., (2018) in dog reported that, the diameter of collagen fibers in the caudal regions was thicker than in the rostral and central regions.

Interestingly, the distribution of elastic fibers in the sheep disc was similar to that reported in humans (Clement, 2006) and minipig (Vapniarsky et al., 2017) which increased in the anterior and posterior regions so as to support the disc. Also, the elastic fibers within the disc provide it with viscoelastic properties to act as a stress absorber and distributor (Chin et al., 1996, Tanaka et al., 2003, Beek et al., 2001).

\section{Conclusion:-}

The full morphological description of TMJ proposed as an anatomical reference of the TMJ of the sheep. Easily surgical approach to TMJ renders the sheep more suitable for procedural surgical training. The anatomical similarities to the human TMJ in; relations, size, shape, structure and position of the disc. All this renders the sheep not only suitable but more preferable as a large animal model over other animal models.

\section{Acknowledgment:-}

The author extends her appreciation and grateful to dr. Eman I El-behery Lecturer of Anatomy and Embryology, Faculty of Veterinary Medicine, Zagazig University, Egypt for her kind support during this study.

\section{Conflict of interests:}

The author declares that she has no conflict of interests.
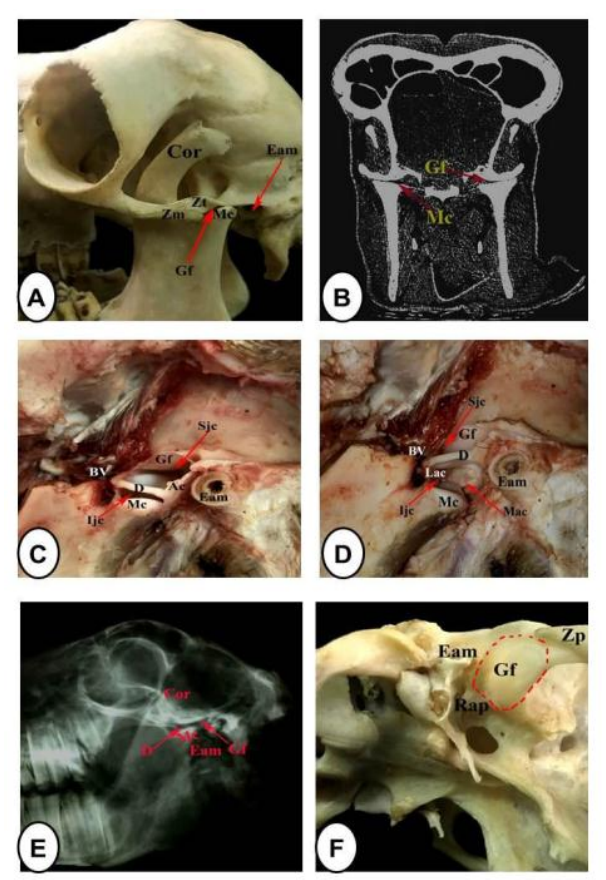

Figure 1:: A Photomacrograph of sheep's head (A) posterior part of skull lateral view, (B) Computed tomography, coronal section (C) and (D) with TMJ dissected lateral view,(E) TMJ radiography, lateral view, (F) Skull 
ventrolateral view, showing; coronoid process (Cor.), zygomatic process of malar ( $\mathrm{Zm})$, zygomatic process of temporal (Zt), Mandibular condyle (Mc), External acoustic meatus (Eam) ,Glenoid fossa (Gf),dick (D), Superior joint cavity (Sjc),Inferior joint cavity (Ijc), Articular capsule (Ac), Medial articular capsule (Mac), Lateral articular capsule (Lac), Blood vessels (Bv), retroarticular process (Rap), and zygomatic process ( $\mathrm{Zp}$ ).
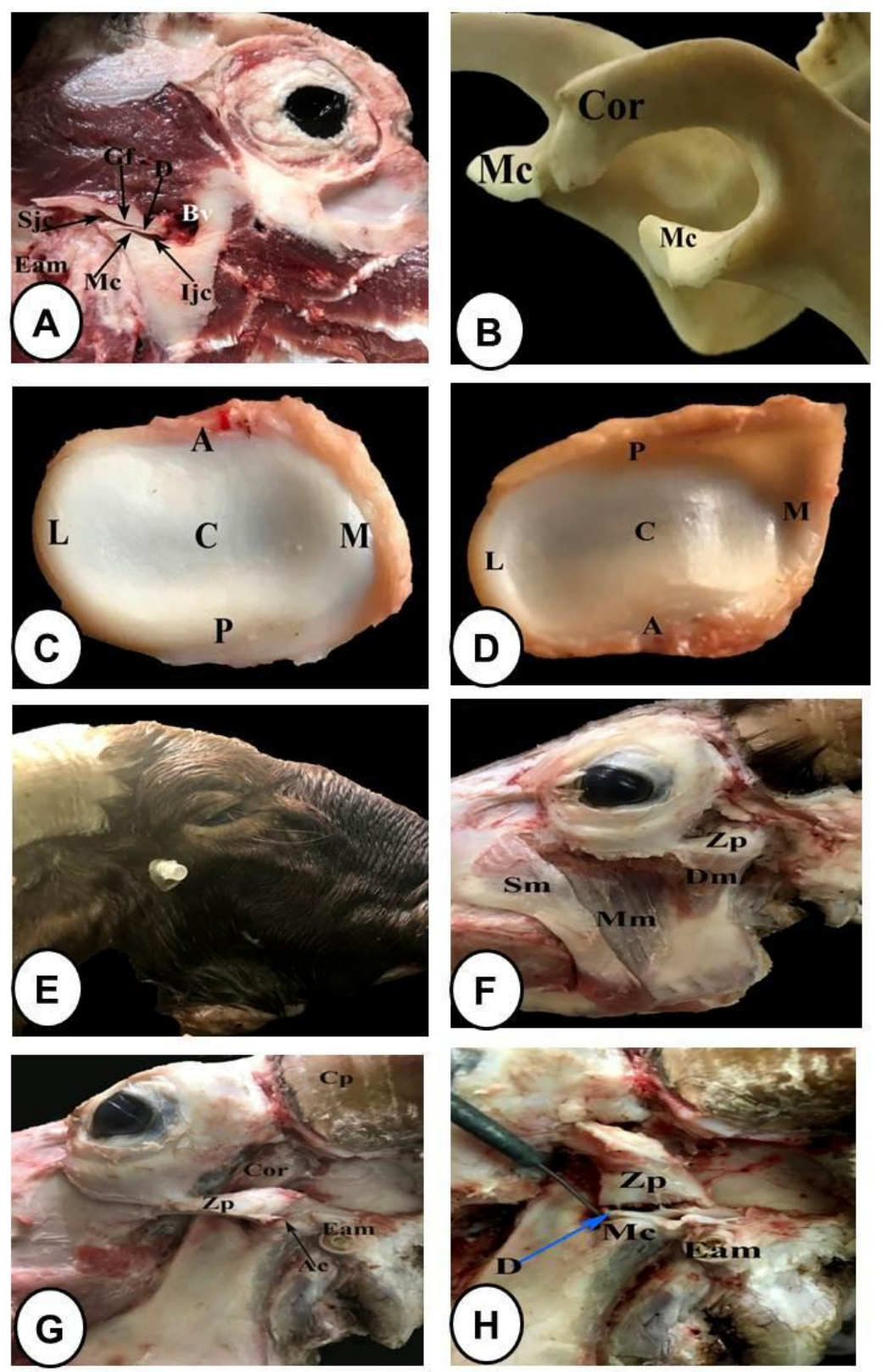

Figure 2:A Photomacrographs of sheep (A) head sagittal section lateral view,(B) mandibule caudolateral view, (C) disk dorsal view (D) disk ventral view, (E) head lateral view with accurate site of injection of TMJ (Needle), (F-H) steps of dissection for surgical approach of TMJ lateral views, showing; Mandibular condyle (Mc), Glenoid fossa (Gf), dick (D), Superior joint cavity (Sjc), Inferior joint cavity (Ijc), External acoustic meatus (Eam), blood vessels $(\mathrm{Bv})$, coronoid process (Cor.), anterior(A), posterior $(\mathrm{P})$, lateral $(\mathrm{L})$, medial $(\mathrm{M})$, central $(\mathrm{C})$, zygomatic process $(\mathrm{Zp})$, superficial $(\mathrm{Sm})$, middle $(\mathrm{Mm})$ and deep $(\mathrm{Dm})$ masseter muscles, corunal process $(\mathrm{Cp})$ and Articular capsule (Ac). 


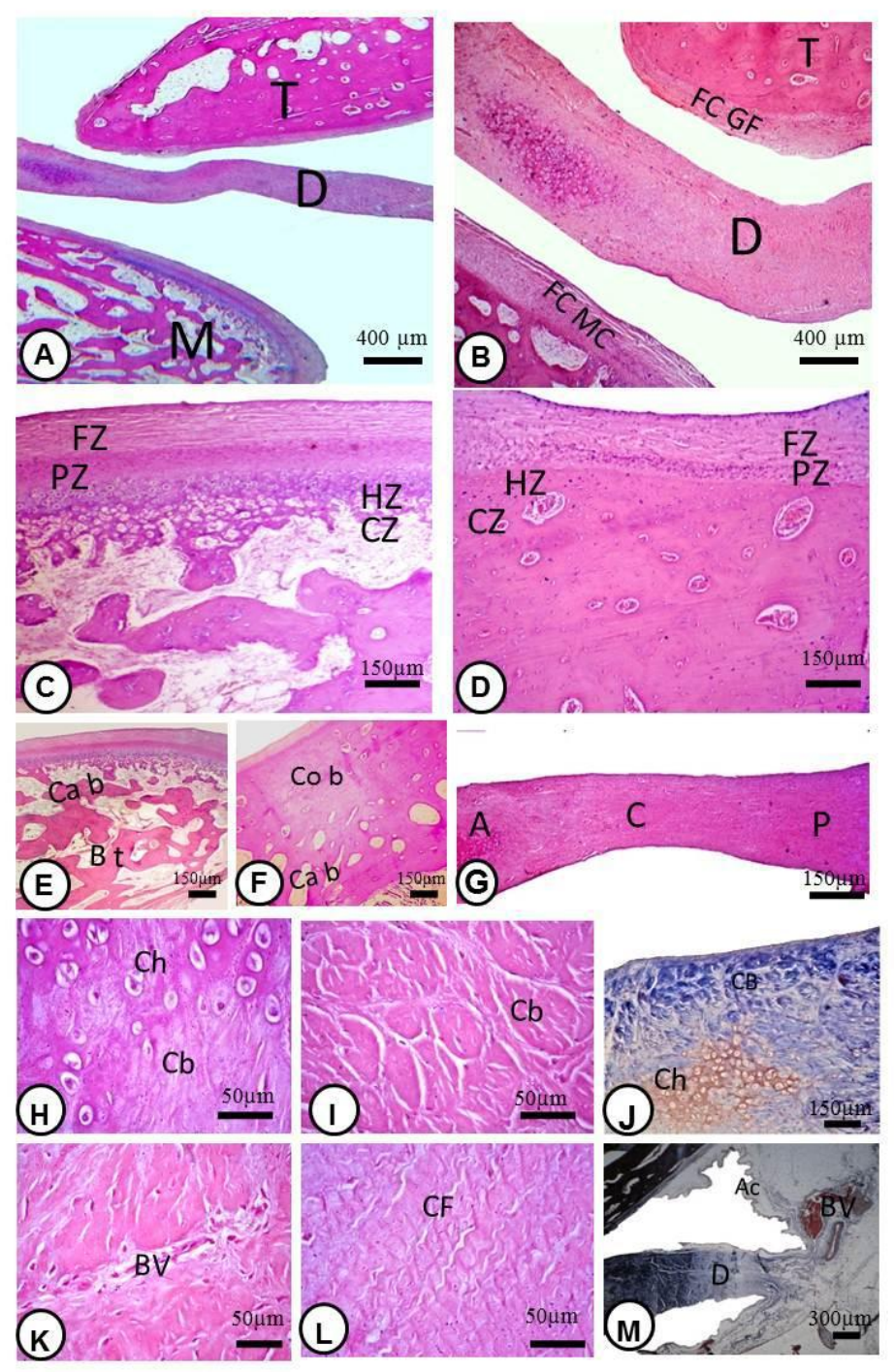

Figure 3: Photomicrographs of sheep TMJ H\&E stain (A-I, K-L) and Crossmon's trichrome stain (J,M): (A) and (B) components of TMJ scale bar $400 \mu \mathrm{m}$, fibrocartilaginous layer of mandibular condyle(C) and glenoid fossa (D) scale bar $150 \mu \mathrm{m}$, bones of mandible(E) and glenoid fossa (F) scale bar $150 \mu \mathrm{m},(\mathrm{G})$ Parts of the disc scale bar

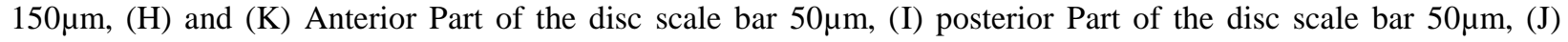

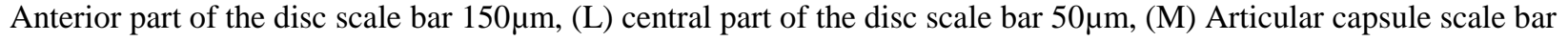
$300 \mu \mathrm{m}$; showing: Temporal bone (T), Mandibule (M), disc (D), Fibrocartilaginous layer of glenoid fossa (FC GF), Fibrocartilaginous layer of mandibular condyle (FC MC), Fibrous (FZ), Proliferative (PZ), Hypertrophic (HZ), Calcified zones(CZ), Bone Trabeculae (Bt), cancellous bone (Ca B), Compact bone (Cb), Anterior (A), Center $(\mathrm{C})$, Posterior $(\mathrm{P})$, Collagen bundles $(\mathrm{Cb})$, Collagen fibers $(\mathrm{CF})$, Chondrocyte $(\mathrm{Ch})$, Blood vessels $(\mathrm{Bv})$, Articular capsule (Ac). 

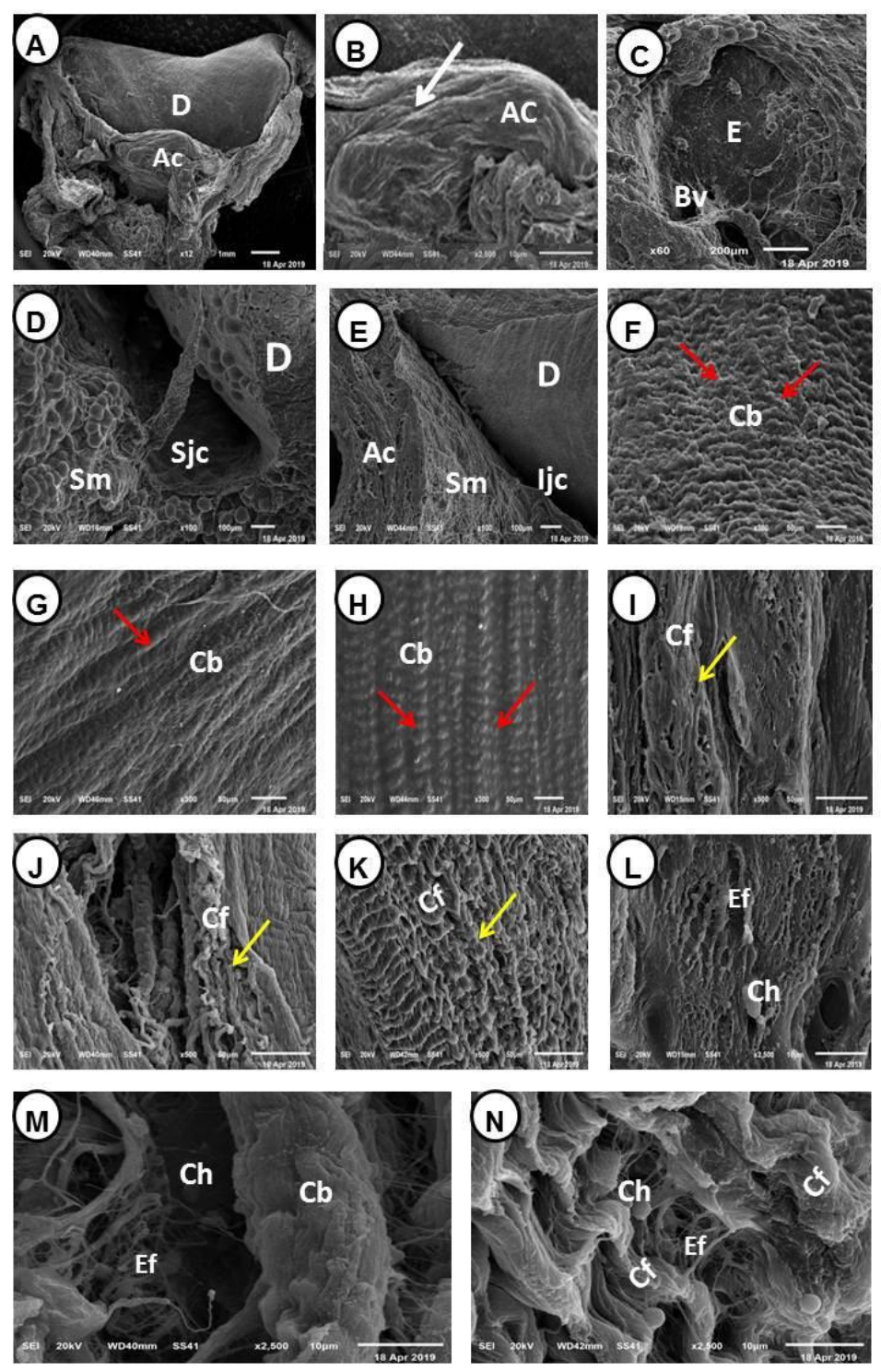

Figure 4:Scanning electron micrographs of sheep's (A-E) disc and attached articular capsule, the dorsal view of disc anteriorly $(F)$, parallel antero-posterior centrally $(G)$, perpendicular posteriorly $(H)$, Cross section view to note the different thickness of fibers which are significantly thicker diameters in the anterior (I), and posterior (K) than central (J) regions. Elastic fiber networks between the collagen in the different regions of the disc (L-N); showing disc (D), articular capsule (Ac) synovial membrane (Sm), Blood vessels (Bv), folded articular capsule white arrow, superior joint cavity ( $\mathrm{Sjc}$ ), Inferior joint cavity (Ijc), Collagen fibers $(\mathrm{Cf})$, Collagen bundles $(\mathrm{Cb})$, corrugated folds (red arrows), thickness of collagen fiber (yellow arrows), Elastic fibers (Ef), Erythrocytes (E).

\section{References:-}

1. Almarza, A.J., Brown, B.N., Arzi, B., Ângelo, D. F., Chung, W., Badylak, S. F. and Detamore, M. (2018): Preclinical Animal Models for Temporomandibular Joint Tissue Engineering. Tissue Engineering Part B: Reviews, 24(3): 171-178.

2. Almarza, A.J. and Chung, W. (2019): Regenerative medicine approaches in large animal models for the temporomandibular joint meniscus. Stomatological Dis Sci., 3:6.

3. Ângelo, D.F., Morouço, P., Alves, N., Viana, T., Santos, F., González, R., Monje, F., Macias, D., Carrapiço, B., Sousa, R., Gonçalves, S.C., Salvado, F., Peleteiro, C. and Pinho, M. (2016): Animal model for temporomandibular joint research: morphological, histological and biomechanical characterization of the Black Merino sheep joint disc. Morphologie, 100 (331):223-233. 
4. Bancroft, J.D. (2008): Theory and practice of histological techniques. 6th ed. Elsevier.

5. Beek, M., Aarnts, M.P., Koolstra, J.H., Feilzer, A.J. and van Eijden, T.M. (2001): Dynamic properties of the human temporomandibular joint disc. J Dent Res., 80(3):876-880. PMID: 11379888

6. Bermejo, A., Gonzalez, O. and Gonzalez, J.M. (1993): The pig as an animal model for experimentation on the temporomandibular articular complex. Oral Surg Oral Med Oral Patho.,75(1):18-23.

7. Bifano, C., Hubbard, G. and Ehler, W. (1994): A comparison of the form and function of the human, monkey, and goat temporomandibular joint. J Oral Maxillofac Surg., 52:272-275. [PubMed] [Google Scholar]

8. Bosanquet, A. and Goss, A.N. (1987): The sheep as a model for temporomandibular joint surgery. Int J Oral Maxillofac Surg., 16:600-603.

9. Bosanquet, A., Ishimaru, J. and Goss, A.N.(1991): Effect of experimental disc perforation in sheep temporomandibular joints. Int J Oral Maxillofac Surg., 20(3):177-181.

10. Chin, L.P., Aker, F.D. and Zarrinnia, K. (1996): The viscoelastic properties of the human temporomandibular joint disc. J Oral Maxillofac Surg., 54(3):315-318-319. PMID: 8600239.

11. Clement, C., Bravetti, P., Plenat, F., Foliguet, B., Haddioui, A.E., Gaudy, J.F. and Weissenbach, M. (2006): Quantitative analysis of the elastic fibers in the human temporomandibular articular disc and its attachments. Int J Oral Maxillofac Surg., 35, 1120.

12. Cornish, R.J. (2005): Histomorphometric analysis of the tempromandibular joint condyle in young and mature sheep. Master thesis of science in Dentistry, University of Adelaide, South Australia.

13. de Moraes, L.O., Lodi, F.R., Gomes, T.S., Marques, S.R., Oshima, C.T., Lancellotti, C.L., Rodríguez-Vázquez, J.F., Mérida-Velasco, J.R. and Alonso, L.G. (2011): Immunohistochemical expression of types I and III collagen antibodies in the temporomandibular joint disc of human fetuses. European Journal of Histochemistry, 55 (3): 129-132.

14. Dolwick, F.M. (1995): Intra-articular disc displacement. Part I. Its questionable role in temporomandibular joint pathology. J Oral Maxillofac Surg., 53:1069.

15. Gillbe, G. V. (1973): A comparison of the disc in the craniomandibular joint of three mammals. Acta Anat., 86 (3): 394-409.

16. Goss, A.N., Jones, R.H.B., Kurita, K. and Handa, Y. (1992): The sheep Tempromandibular Joint model: A Japanese/ Australian Investigation. Hospital Dentistry and oral Maxillofacial Surgery. (Tokyo), 4: 58-60.

17. Herring, S.W. (2003): TMJ anatomy and animal models. J Musculoskel Neuron Interact., 2003; 3(4):391-394.

18. Ide, Y., Nakazawa, K. and Garcia, L.T. (1991): Anatomical atlas of the temporomandibular joint. Quintessence Publ. Co., Chicago: 116.

19. Ingawalé, S. and Goswami, T. (2009): Temporomandibular joint: disorders, treatments, and biomechanics. Ann Biomed Eng., 37(5):976-96. doi: 10.1007/s10439-009-9659-4.

20. Ishimaru, J. and Goss, A.N. (1992): A model for osteoarthritis of the temporomandibular joint. J Oral MaxillofacSurg.50:1191-1195.

21. Jung, C.K. (2014): Articular Cartilage Histology and Physiology in Techniques in Cartilage Repair Surgery. Shetty AA, Kim S, Nakamura N, Brittberg M. Springer Heidelberg New York Dordrecht London. (Chapter 2: 32-36).

22. Kabak, M. (2002): Akkaraman koyununda articulation temporomandibularis ve çiğneme kaslarının anatomisi. Ankara. Univ. Vet. Fak. Derg., 49: 7-11.

23. Kabak, M., Tütüncü, S., Demirci, B., Onuk, B. (2014): Anatomy of the Temporomandibular Joint and Masticatory Muscles in Roe Deer, Capreolus capreolus. Pakistan J. Zool., 46(5):1363-1370.

24. Lin, W.A., Vapniarsky, N., Cissell, D.D., Verstraete, F.J.M., Lin, C.H., Hatcher, D.C. and Arzi, B. (2018): The Temporomandibular Joint of the Domestic Dog (Canis lupus familiaris) in Health and Disease. J. Comp. Path., 161: 55-67.

25. Minarelli, A.M., Del Santo Junior, M. and Liberti, E.A. (1997): The structure of the human temporomandibular joint disc: a scanning electron microscopy study. J Orofac Pain, 11(2): 95-100.

26. Ness, G.M. (2011): Arthroplasty and discectomy of the temporomandibular joint. Atlas Oral Maxillofac Surg Clin North Am., 19(2): 177-187.

27. Ogi, N., Kurita, K., Ishimaru, J.I., Goss, A.N. (1999): Short-term effect of the use of a frozen-stored disc allograft for repair of the osteoarthritic sheep temporomandibular joint: a preliminary report. J Oral Maxillofac Surg., 57(2):139-44-5. PMID: 9973121

28. Onwuama, K.T., Salami, S.O., Ali, O. and Nzalak, J.O. (2012): Effect of different methods of bone preparation on the skeleton of the african giant pouched rat (Cricetomys gambianus). Int. J. Morphol., 30(2):425-427.

29. Piette, E. (1993): Anatomy of the human temporomandibular joint. An updated comprehensive review. Acta Stomatol Belg., 90(2):103-127. 
30. Porto, G.G., Vasconcelos, B.C., Andrade, E.S. and Silva-Junior, V.A. (2010): Comparison between human and rat TMJ: anatomic and histopathologic features. Acta Cir Bras., 25: 290-293.

31. Purcell, P., Jheon, A., Vivero, M.P., Rahimi, H., Joo, A. and Klein, O.D. (2012): Spry1 and Spry2 are essential for development of thetemporomandibular joint. Journal of Dental Research, 91(4): 387-393.

32. Rashed, F. (2015): A Comparative Study of the Dentition and Temporomandibular Joint Anatomy and Histology Adult Dogs. Biol syst Open Access, 4:2. DOI: 10.4172/2329-6577.1000147.

33. Rodríguez, M.J., Agut, A., Gil, F. and Latorre, R. (2006): Anatomy of the equine temporomandibular joint: study by gross dissection, vascular injection and section. Equine Vet. J., 38(2):143-147.

34. Sabú, C., Carvalho de Moraes, L.O., de Quadros Uzêda-Gonzalez, S., Marques, S.R., Vretos, C., Aguillera, A.H., Sasso Scarpati, G.R.S., Correa Harb, L.J., Sassoli Fazan, V.P., Oshima, C., Fernandes Junior, J.A., Smith, R.L. and Garcia Alonso, L. (2006): Ultrastructural evidence of vascularization of the central zone of the temporomandibular joint disc in human fetus. Eur J Anat., 10 (2): 45-48.

35. Scapino, R.P., Canham, P.B., Finlay, H.M. and Mills, D.K. (1996): The behaviour of collagen fibers in stress relaxation and stress distribution in the jaw-joint disc of rabbits. Arch Oral Biol., 41:1039-1052. [PubMed] [Google Scholar]

36. Sindelar, B.J., Edwards, S. and Herring, S.W. (2002): Morphologic changes in the TMJ following splint wear. Anat Rec., 266:167-176.

37. Smith, K.K. (2001): The evolution of mammalian development. Bull Mus Comp Zool., 156:119-135.

38. Ström, D., Sato, H. and Magnusson, B. (1996): Microanatomy of the moose temporomandibular joint (Alces alces; Linnaeus, 1758). Eur. J. Oral. Sci., 104 (5-6):589-594.

39. Tahmasebi-Sarvestani, A. (1997): Innervations of the tempromandibular joint, an experimental animal model using Australian Merino Sheep. Doctor of Philosophy thesis, Department of Anatomical Science, University of Adelaide, South Australia.

40. Tanaka, E., Detamore, M. S., Tanimoto, K. and Kawai, N. (2008): Lubrication of the temporomandibular joint. Annals of Biomedical Engineering, 36(1):14-29.

41. Tanaka, E. and van Eijden, T, (2003): Biomechanical behavior of the temporomandibular joint disc. Crit Rev Oral Biol Med., 14(2):138-150. PMID: 12764076

42. Tanne, K., Tanaka, E. and Sakuda, M.(1991): The Elastic Modulus of the Temporomandibular Joint Disc from Adult Dogs. J Dent Res., 70:1545-1548. DOI: 10.1177/00220345910700121401.

43. Vapniarsky, N., Aryaei, A., Arzi, B., Hu, J.C., Hatcher, D.C. and Athanasiou, K.A. (2017): The Yucatan minipig TMJ disc structure-function relationships support its suitability for human comparative studies. Tissue Eng Part C Methods, 23(11): 700-709.

44. Yan, Y.B., Zhang, Y., Gan,Y.H., An, J.G., Li, J.M. and Xiao, E. (2013): Surgical induction of TMJ bony ankylosis in growing sheep and the role of injury severity of the glenoid fossa on the development of bony ankylosis. Journal of Cranio-MaxilloFacial Surgery, 41(6): 476-486. 